\title{
MiR674 inhibits the neuraminidase-stimulated immune response on dendritic cells via down-regulated Mbnl3
}

\author{
Jian Lin ${ }^{1}$, Ya T. Chen ${ }^{1}$, Jing Xia ${ }^{1}$ and Qian Yang ${ }^{2}$ \\ ${ }^{1}$ Life Science College, Nanjing Agricultural University, Weigang, Nanjing, Jiangsu, PR China \\ ${ }^{2}$ College of Veterinary Medicine, Nanjing Agricultural University, Weigang, Jiangsu, PR China \\ Correspondence to: Qian Yang, email: zxbyq@njau.edu.cn \\ Keywords: dendritic cells; neuraminidase; miR-674; immune regulation; virus replication; Immunology and Microbiology Section; \\ Immune response; Immunity \\ Received: November 01,2015 Accepted: May 02,2016 Published: June 06, 2016
}

\section{ABSTRACT}

Neuraminidase (NA), a structural protein of the H9N2 avian influenza virus (H9N2 AIV), can facilitate viral invasion of the upper airway by cleaving the sialic acid moieties on mucin. Dendritic cells (DCs) are major antigen-presenting cells whose immune functions, such as presenting antigens and activating lymphocytes, can be regulated by microRNAs. Here, we studied the molecular mechanism of miRNA-induced repression of immune responses in mouse DCs. First, we screened for and verified the miRNAs induced by NA. Then, we showed that, consistent with the H9N2 virus treatment, the viral NA up-regulated the expression of miR-155, miR-674, and miR499 in DCs; however, unlike H9N2 virus treatment, the presence of NA was associated with reduced expression of miR-181b1. Our results suggest that NA significantly increased DC surface markers CD80 and MHCII and enhanced the ability of activating lymphocytes and secreting cytokines compared with HA, NP and M2. Meanwhile, we found that miR-674 and miR-155 over-expression increased all surface markers of DC. Nevertheless, by inhibiting the expression of miR-674 and miR-155, NA lost the ability to promote DC maturation. Furthermore, we predicted and demonstrated that Pgm2/1, Aldh18a1, Camk1d, and Mbn/3 were the target genes of miR-674. Among them, Mbn/3 interference strongly blocked the mature DCs. Collectively, our data shed new light on the roles of and mechanisms involved in the repression of DCs by miRNAs, which may contribute to efforts to develop a prophylaxis for the influenza virus.

\section{INTRODUCTION}

Frequent outbreaks of reassortant H7N9 avian influenza virus (AIV) caused great threatened to the poultry industry and the public health [1]. Sequencing analyses revealed that six internal genes of H7N9 subtype shared the highest similarity with H9N2 subtype viruses that have circulated in poultry [2]. H9N2 avian influenza virus (AIV) has caused multiple disease pandemics due to its high genetic variability and high rate of recombination with other influenza virus subtypes. Neuraminidase (NA) was an important target for host neutralizing antibodies. Point mutations in the antigenic domains of the NA protein were considered as a way to escape host immune system for viruses [3]. Eckard suggested that NA may be another piece of the influenza vaccine, while European Pharmacopeia suggest to maximize vaccine NA content to induce protective immunity against influenza infection at the same time [4]. So the big question now is how virus protein NA triggered the immune response.

Since dendritic cells (DCs), professional antigen presenting cells, acted as sentinels for monitoring pathogenic microbes, allergens, and pollutants [5]. It is time to propose that if and how NA segment induced the immune activation of DCs. As we known, the uptake of AIV is necessary for DCs triggering innate and subsequent adaptive immune responses to against virus invasion and replication [6]. Studies found that AIV infection can regulate the maturation, the antigen presenting and the cytokines secretion of DCs [7]. Consider NA is a surface glycoprotein of influenza viruses and can induce NA-directed antibody responses that are independently predictive of protection [8]. We need further study how DCs recognized NA and triggered the immune defending for achieving the goals of controlling and eliminating influenza virus effectively.

Vaccination with inactivated vaccine has been a main measurement to prevent avian influenza virus for 
a long time [9, 10]. Given the limitations of the current prevention or treatment of acute influenza, novel therapies are needed. On one hand, RNA interference (RNAi) through microRNAs (miRNAs) is an emerging technology that can suppress virus replication in vitro and in vivo [11]. For example, temporal and specific host miRNA let$7 \mathrm{c}$ has been demonstrated inhibit M1 protein expression of the H1N1 influenza virus in infected human lung epithelial cells [12]. On the other hand, miRNAs affect the development of DCs and their ability to present antigens as well as secret cytokines [13], which may block the infection of AIV. For example, miR451 regulates a subset of pro-inflammatory cytokine to combat with influenza virus in mice DCs [14]. In addition, there are now solid data to suggest a protective role for NA immunity and to prove that contribution of antibody production against neuraminidase to the protection afforded by influenza vaccines $[4,15]$. The purpose of our study was to figure out how miRNAs regulate the immune response of DCs stimulated by virus protein NA.

\section{RESULTS}

\section{Distinctive alteration of miRNAs in response to viral fragment stimulation}

Previously, we studied the influence of H9N2 AIV infection on global RNA expression in mice. We found that nine miRNAs were significantly up-regulated by viral infection and that eight were down-regulated (Supplement 1). To study how H9N2 might control miRNA expression, we studied four segments of the H9N2 virus that were unrelated to replication: neuraminidase (NA), hemagglutinin (HA), matrix protein 2 (M2), and non-structural protein (NS). These genes were cloned into the pcDNA3.1 vector (Supplement 2) and transfected into bone marrow-derived DCs (BMDCs). The expression of select miRNAs was examined by quantitative PCR (qPCR). We found that the NA segment significantly
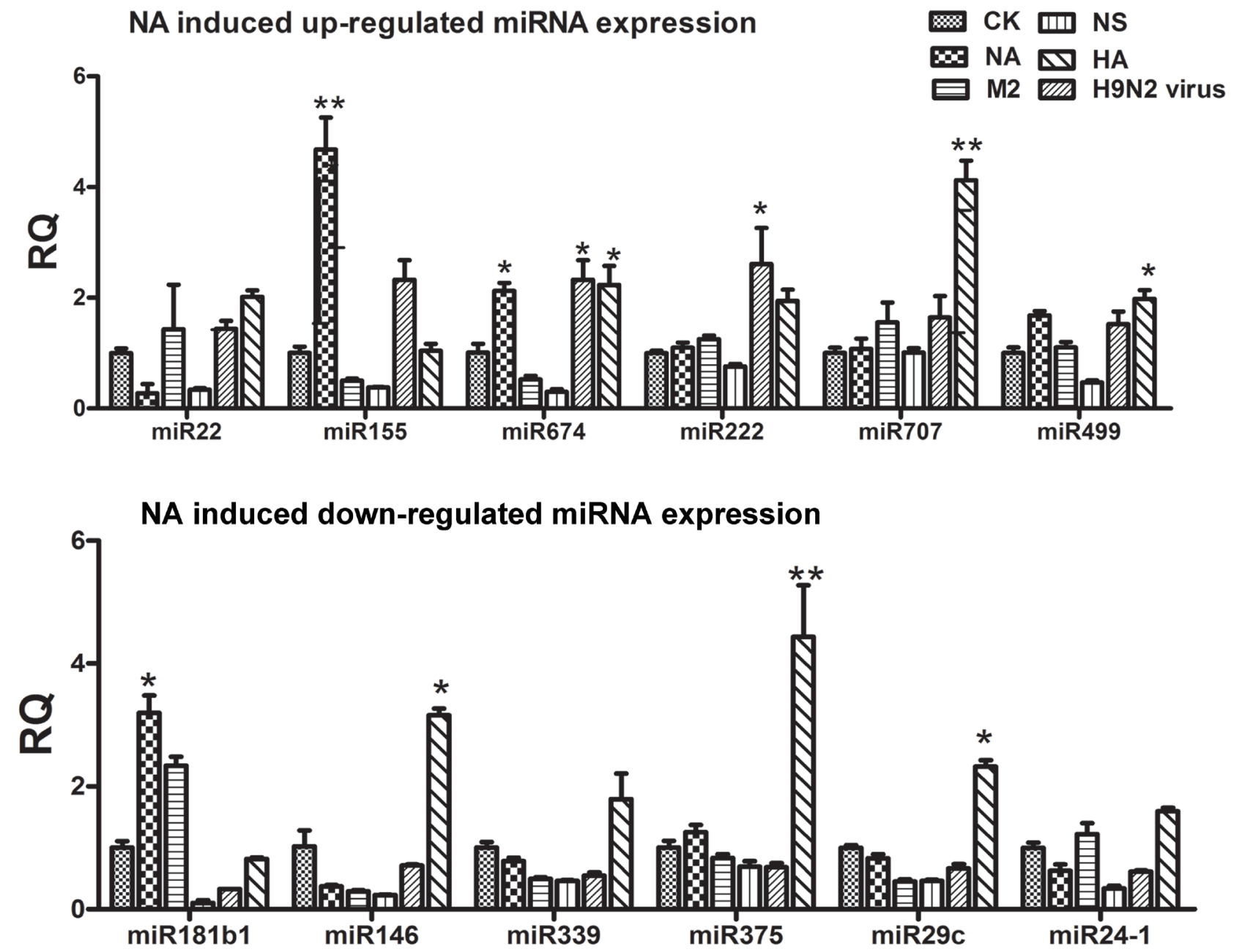

Figure 1: Results of the qPCR analysis of select miRNAs following stimulation by NA, HA, M2, or NS. A. The expression levels of up-regulated miRNAs stimulated by NA, HA, M2, and NS. B. The expression levels of down-regulated miRNAs stimulated by NA, HA, M2, and NS. 
increased the expression of miR-155 and miR-674, whereas the HA segment significantly up-regulated the expression of miR-707, miR-674, and miR-499 (Figure 1A). For those miRNAs down-regulated by H9N2, the NA segment greatly increased the expression of miR181b1; however, unlike the H9N2 virus treatment, the HA treatment significantly rose the expression of miR146, miR375, and miR-29c, (Figure 1B).

\section{Activation of mouse BMDCs by NA}

Next, we investigated how the viral segments affect mouse BMDC functions. There are three standards for evaluating the immune function of BMDCs: phenotypic alterations, the ability to activate $\mathrm{T}$ lymphocytes, and the ability to secrete cytokines [16]. We first examined the phenotypic changes of DCs after stimulation with NA, HA, M2, and NS. Fluorescence-activated cell sorting (FACS) suggested that viral segments NA and M2 significantly enhanced the percentages and mean fluorescent intensity (MFI) of MHCII, as well as of the co-stimulatory molecule CD80 (Figure 2A and 2B). The HA segment up-regulated only CD40, whereas NS had no effect (Figure 2A and 2B). Next, we assessed the ability of DCs to activate lymphocytes and secrete cytokines. As shown in Figures 2C and 2D, DCs treated with NA displayed enhanced stimulation at a ratio of $1: 1$ or 1:5, and higher levels of interleukin (IL)- 6 and tumour necrosis factor (TNF)- $\alpha$ were observed compared with the pcDNA3.1-transfected controls $(P<0.05)$. CD40, CD80/ CD86, and MHCII are characteristic surface markers for fully matured DCs. Additionally, we found that the mRNA expression of Pgm211, Aldh18a1, Camk1d, and Mbnl3, targeted to miR674, decreased significantly when treated with NA (Figure 2E). Myo1d, a target gene of miR155, was greatly down-regulated in the NA group compared with the pcDNA3.1 group (Figure 2E).

\section{Immune function of miR-674 and miR-155 in regulating mice $\mathrm{BMDCs}$}

Recent studies have shown that miRNAs regulate the immune responses of BMDCs [17]. Given that the H9N2 virus and NA segment significantly up-regulated the expression of miR-155 and miR-674 (Figure 1A), we examined the functions of these miRNAs in BMDCs. miRNA over-expression vectors were constructed and validated, as shown in Supplement 3. FACS results indicated that miR-674 and miR-155 significantly increased the percentage and MFI of CD40-positive cells (Figure 3A and 3B) compared with the pSilencer group. Interestingly, miR-181b1 increased the percentage and MFI of MHCII-positive cells. Moreover, overabundance of miR-155 and miR-674 promoted lymphocyte proliferation at a ratio of $1: 1$ and 1:5 respectively (Figure
$3 C)$. Furthermore, we found that miR-674 increased the expression of IL6, IL-12, and TNF- $\alpha$, whereas miR-155 induced IL-6, IL-10, and TNF- $\alpha$ production (Figure 3D). Additionally, we found that the mRNA expression of Myo1d, Pgm211, Aldh18a1, Camk1d, and Mbnl3, targeted to miR155 or miR674, were significantly decreased in miR155 and miR674 over-expression groups compared with the pSilencer4.1 control group (Figure 3E).

\section{Inhibition of endogenous miRNA function blocked NA-induced phenotypic alterations in BMDCs}

Previous studies have demonstrated that NA and miR-674 or miR-155 have similar effects on BMDCs. Thus, we investigated whether the NA-induced changes in DCs are mediated by miRNAs. To test this hypothesis, miRNA inhibitors were designed and added to DCs to repress endogenous miRNAs prior to NA transfection. FACS revealed that the inhibition of endogenous miR-674 or miR-155 decreased the expression of co-stimulatory molecules (CD80/CD86 and CD40) and MHCII, which was induced by NA. Moreover, NA did not increase the MFI of CD40- and MHCII-positive cells when miR-499 or miR-181b1was inhibited $(\mathrm{P}<0.05)$ (Figure 4A and 4B).

\section{MiR-674 and miR-155 target prediction and validation}

miRNAs exert their functions by causing translational repression or mRNA degradation. To study the mechanism of action by miR-155 and miR-674, we used Miranda and Targetscan to predict miR-155 and miR674 target genes, considering only the overlap of both algorithms, and further matched the prediction based on our previous microarray data. Finally, we selected Myod1 targeted to miR-155 and Pgm2l1, Entpd6, Aldh18al, Camk1d, Igf1r, or Mbnl3 targeted to miR-674 for further testing. Luciferase reporter assays showed that Pgm2l1, Aldh18a1, Camk1d, and Mbnl3 groups significantly decreased luciferase activity $(\mathrm{P}<0.05)$, whereas Entpd6 and Igflr groups non-significantly reduced luciferase activity (Figure 4D). Similar results were observed in the Myold group; luciferase activity decreased markedly once we added miR-155, which did not occur in the Myoldmuted group (Figure 4C).

\section{Silenced endogenous target gene blocked NA- induced phenotypic alterations in BMDCs}

Our study demonstrated that Myold, Camkld, and Mbnl3 were the target gene of miR-155 and miR-674. To determine whether the knockdown of Myold, Camkld, and Mbnl3 had any gross effect on the immune function of BMDCs, we assessed the phenotypic alteration of DCs 

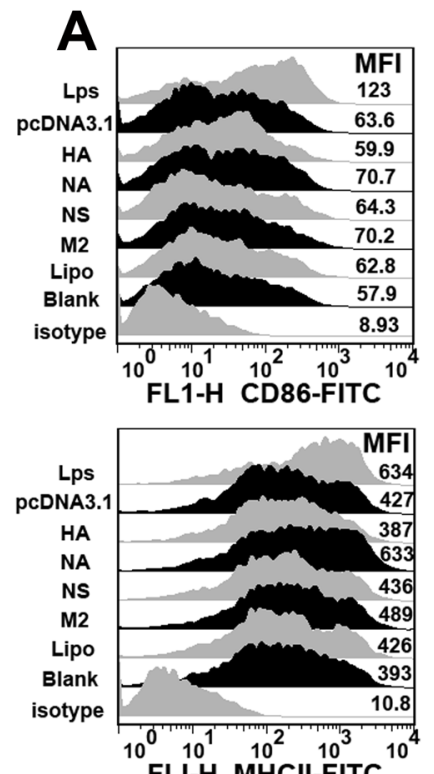

FLI-H MHCII-FITC


E
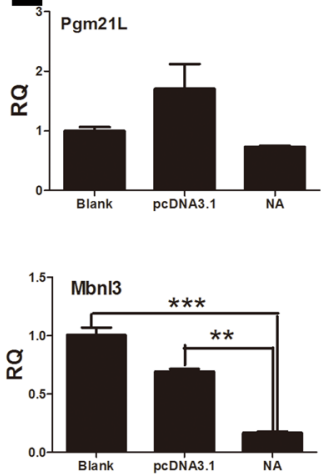

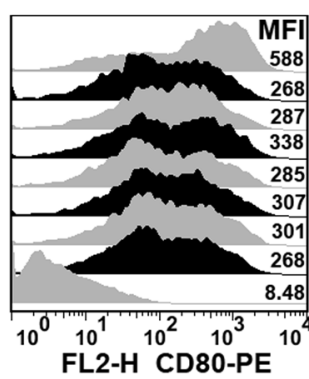

B
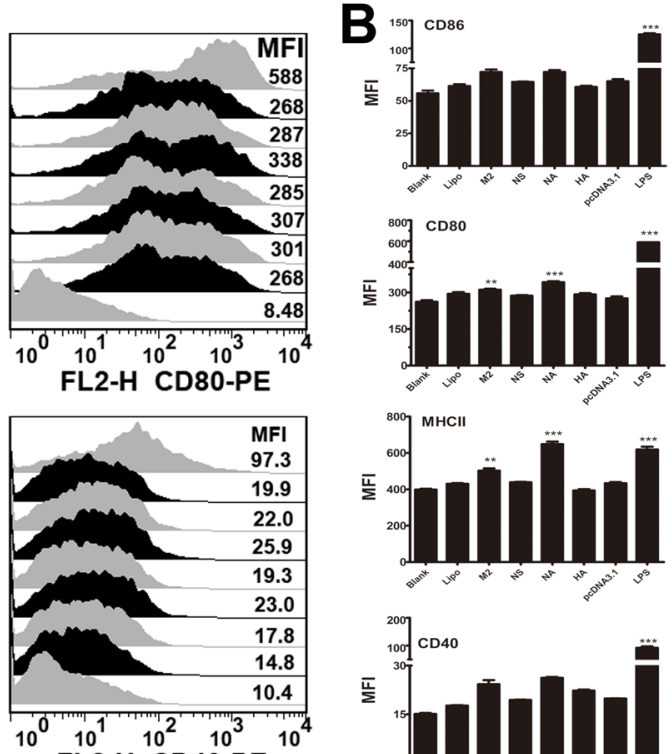

FL2-H CD40-PE

D

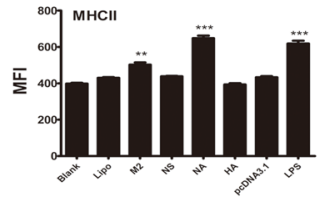

$\left.\right|_{0} ^{200}$
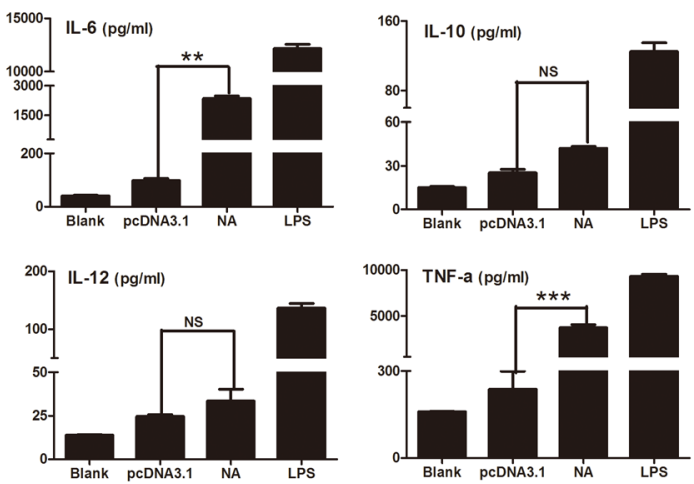

Figure 2: Immune activation of BMDCs stimulated by NA. A. Flow cytometric analysis of the phenotypic alterations in DCs stimulated with NA, HA, M2, or NS (i.e. the expressions of CD40, CD80/86, and MHCII on BMDCs stimulated with NA, HA, M2, or NS). Positive control, $1 \mu \mathrm{g} / \mathrm{ml}$ LPS. B. The MFI of CD40, CD80/86, and MHCII. C. NA-stimulated BMDCs stimulated the proliferation of naive $\mathrm{T}$ cells in mixed-lymphocyte reactions (MLR). The stimulator cells were BMDCs stimulated with or without NA, pcDNA3.1, or LPS at $37^{\circ} \mathrm{C}$ for $24 \mathrm{~h}$. All experiments were performed at least in triplicate. Significant differences between the treated and control groups are expressed as $* P<0.05$ or $* * P<0.01$. D. Cytokine release from NA-stimulated BMDCs was measured by enzyme-linked immunosorbent assays (ELISAs). Data for IL-6, IL-10, IL-12, and TNF- $\alpha$ are shown as the mean \pm standard deviation (SD) of three samples. $* * P<0.01,{ }^{*} P$ $<0.05$ compared with the LPS-only group. All results are representative of three independent experiments. E. Results of the qPCR analysis of select target genes (Myold, Pgm2l1, Aldh18al, Camk1d, and Mbnl3) following stimulation by NA. 

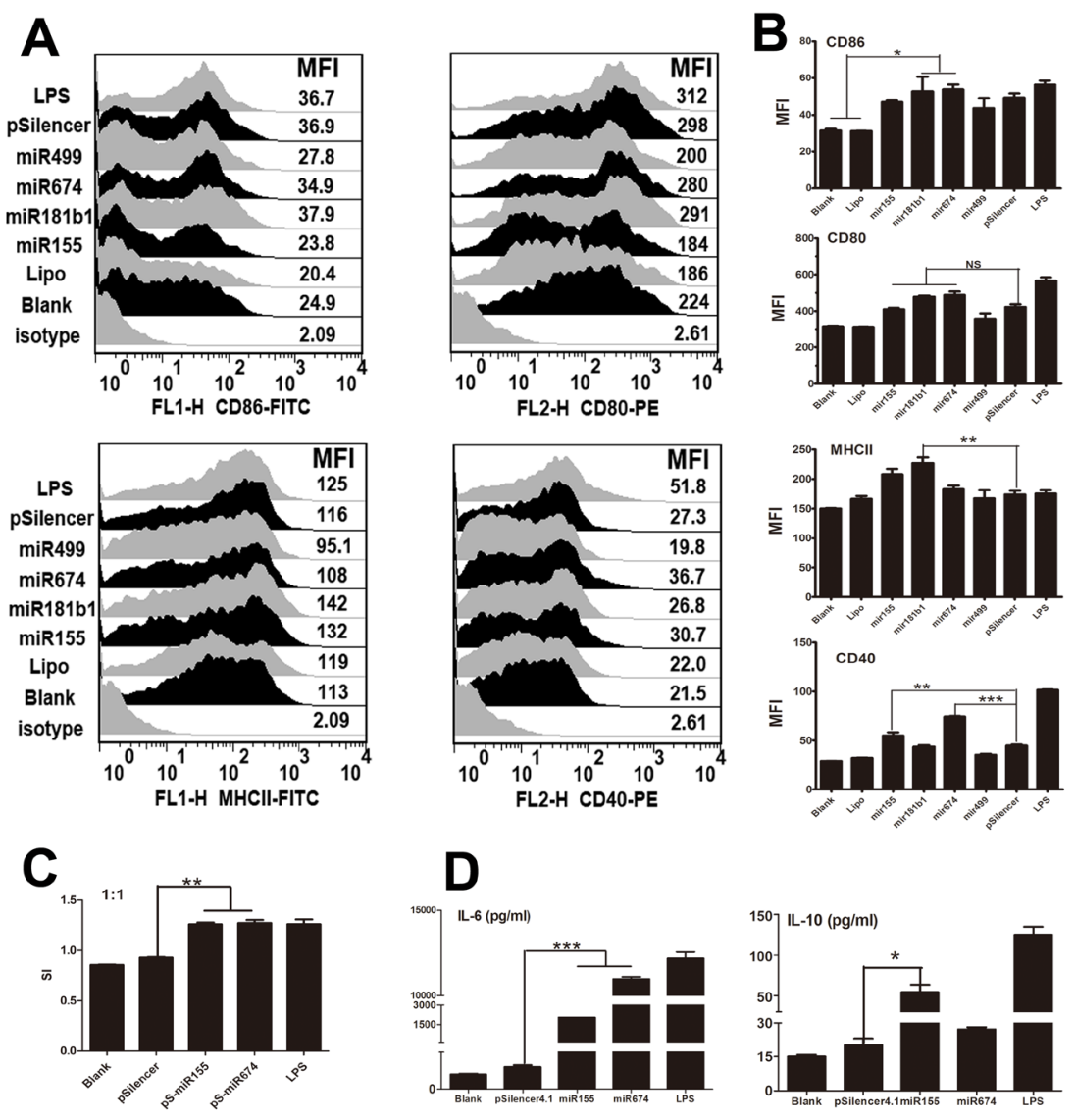

D
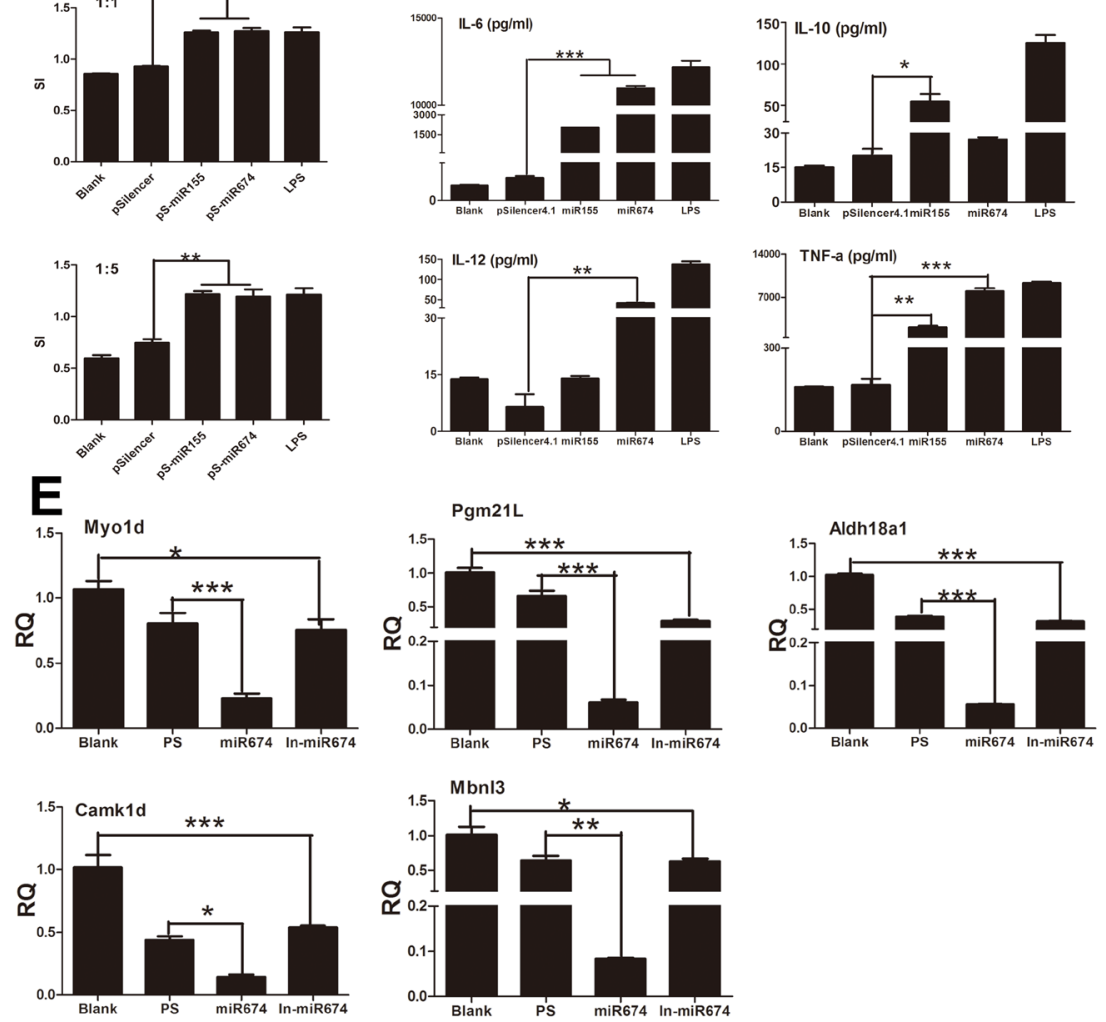

Figure 3: Immune function of BMDCs stimulated by miRNAs. A. Flow cytometric analysis of the phenotypic alterations in DCs stimulated by miR155, miR499, miR375, miR674, or miR181b1 (i.e. the expressions of CD40, CD80/86, and MHCII on BMDCs stimulated by miRNAs). Positive control, $1 \mu \mathrm{g} / \mathrm{ml}$ LPS. B. The MFI of CD40, CD80/86, and MHCII. C. miR-155- or miR-67-stimulated BMDCs stimulated the proliferation of naive T cells in MLR. The stimulator cells were BMDCs stimulated with or without miR29c, pSilencer, or LPS at $37^{\circ} \mathrm{C}$ for $24 \mathrm{~h}$. All experiments were performed at least in triplicate. Significant differences between the treated and control groups are expressed as $* P<0.05$ or $* * P<0.01$. D. Cytokine release from BMDCs stimulated by miR-155 or miR-674 measured by ELISA. Data for IL-6, IL-10, IL-12, and TNF- $\alpha$ are shown as the mean \pm SD of three samples. ${ }^{* *} P<0.01, * P<0.05$ compared with the LPS-only group. All results are representative of three independent experiments. E. Results of the qPCR analysis of select target genes Myold, Pgm2l1, Aldh18al, Camk1d, and Mbnl3) following stimulation by miR155 or miR674. 



C

FL2-H CD40-PE

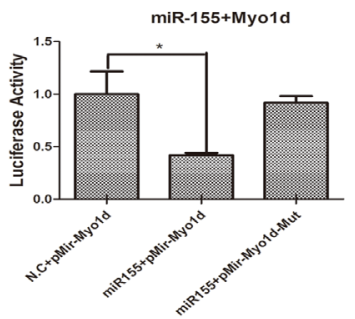

miR-155+Myo10

$5 '$...CAUCAAGAGCAAUAAATCTAGCAA......MYOID Mut.

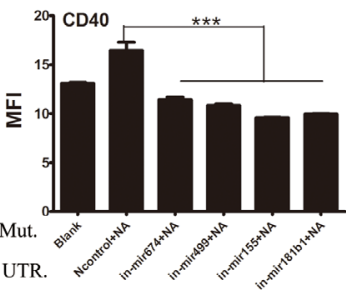

||||||||

3' $\quad$ UGgGgauaguguUAaUdGUAAUU.......mmu-miR-155

D
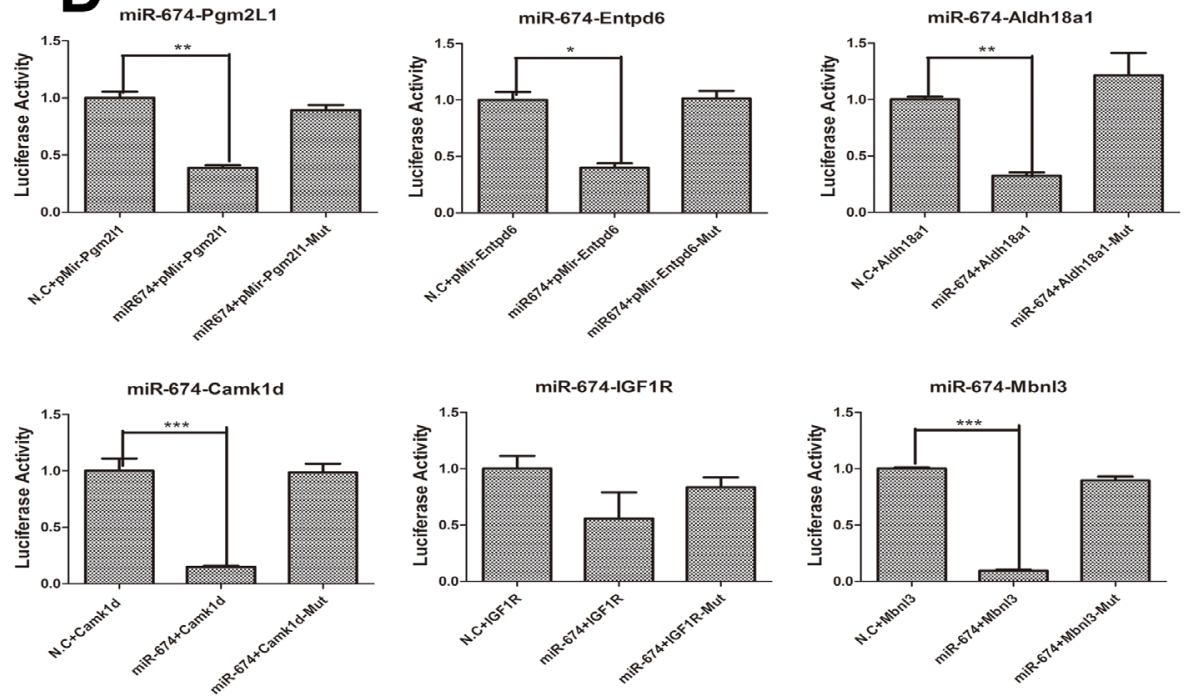

Figure 4: Results of miRNA inhibitor treatment and dual luciferase reporter assays for miR155, miR674, and their target genes. A. The expressions of CD40, CD80/86, and MHCII on BMDCs when miR-155, miR-674, miR-499, and miR-181b1were inhibited (100 $\mu \mathrm{g} / \mathrm{ml}$ inhibitor for each miRNA). B. The MFI data for CD40, CD80/86, and MHCII. C. The map position of gene Myold and its mutated site targeted to miR155, and the dual luciferase assay results for miR-155 and its target genes (level of significance: $P<$ 0.05 or $P<0.01$ ). D. Dual luciferase assay results for miR-674 and its target genes (level significance: $P<0.05$ or $P<0.01$ ). 
when these genes were knocked down or over-expressed. On one hand, qPCR results revealed that the mRNA levels of all three genes were reduced after siRNAs lentivirus vector transfection (Figure $5 \mathrm{C}$ ). Of these siRNAs, the greatest decrease in Myold mRNA levels was achieved using siMyold-1, whereas siCamk1d-9 and siMbnl3-15 significantly reduced their mRNA expression levels compared with the negative control. FACS results consistently suggested that BMDCs lose the ability to raise their surface marker CD86 and MHCII when Mbnl3 or Myold genes were silenced (Figure 5A and 5B). Furthermore, the inhibitory effect of silencing Mbnl3 or Myold genes did not recover any further even when their mirror miRNAs were added to DCs (Figure 5A and 5B). These data strongly suggested that Mbnl3 or Myold may be the next target for combating influenza virus. On the other hand, FACS result suggested that the overexpression of Mbnl3 hugely increased the surface marker CD86 and MHCII, whilst the over expressed Mbnl3 had no effect on CD40 and CD80 (Supplement 9).

\section{Effects of NA, miR-674, and miR-155 on signalling pathways}

Previously, we demonstrated that H9N2 AIV activates interferon (IFN) regulatory factor (IRF)-7 and TNF receptor-associated factor (TRAF)-6. Thus, we continued to explore the signalling pathways that may be activated by NA and miR155 or miR674. As we known, mitogen-activated protein kinase (MAPK) pathways exist in all eukaryotes and control a wide range of cellular processes, such as proliferation, differentiation, and survival. In this study, we found that NA segment induced the activity of IkBa and P38 signal pathway by phosphorylating it. Whilst, NA stimulation has no effect or decreased the JNK and ERK signal pathways (Supplement 7). Moreover, we found that miR-155 overabundance activated both IkBa and P38 signalling pathways, while miR-674 over-expression group just activated the P38 signal pathway(Supplement 7). Furthermore, we found that NA stimulation significantly down-regulated the expression of IRF-3, TRAF-3, and-6. Additionally, the expression of IRF-3 and TRAF-3 were slightly decreased in miR-674 group, compared with blank group. In terms of the miR-155 over-expression group, the levels of IRF7 and TRAF3/6 increased slightly compared with the decreased IRF-3 expression (Supplement 6). These results not only indicate that the activation of P38 pathway may involved in the regulation of the DC function when NA or miR-674 stimulation, but also suggested that IRF-3 might be involved in the regulating of NA-stimulated DC immune function.

\section{Effects of miR674 or miR155 inhibition or over- expression on virus replication}

Considering that miR674 and miR155 significantly affected NA-induced DC function, we evaluated whether miR674 or miR155 could influence the replication of H9N2 AIV. We generated standard curves for PB1 and GAPDH (Figure 6A and 6B) and then conducted absolute qPCR. Our results showed that the inhibition of miR674 or miR155 significantly up-regulated viral replication at 6 and $24 \mathrm{~h}$, whereas the over-expression of miR674 or miR155 repressed viral replication (Figure 6C and 6D).

\section{DISCUSSION}

The interactions between cellular factors, such as miRNAs and H9N2 AIV, are important for AIV infection and replication. In this report, we found that the NA segment of H9N2 AIV modulated the immune function of DCs by adjusting miR-155 and miR-674 expression. NA, an envelope glycoprotein on the surface of the influenza virus, catalyses the cleavage of sialic acids on glycoproteins from virus-infected cells and enables virus release $[18,19]$. Studies have demonstrated that low NA enzyme activity renders virus release from infected cells inefficient and leaves progeny viruses gathered on the cell surface $[19,20]$. DCs represent a central element in the generation and maintenance of immune responses [21]. Our study suggested that the immune functions of DCs, including phenotypic alteration, T lymphocyte activation, and cytokine secretion, were greatly stimulated by NA. Additionally, we identified four cellular miRNAs (miR155, miR-674, miR-499, and miR-181b1) that were significantly up-regulated by NA. Among them, miR-155 and miR-674 were shown to support the ability of DCs to activate lymphocytes.

MiR-155 shapes the balance between tolerance and immunity, which plays a critical role in immunity and viral infections [22]. miR-155 is involved in fine-tuning the regulation of lymphocyte subsets, such as B cells, $\mathrm{CD}^{+}$, and $\mathrm{CD}^{+} \mathrm{T}$ cells including $\mathrm{T}$ helper type 1 (Th1), Th2, Th17, and regulatory T cells [23]. Considering that the level of miR-155 is correlated with immune function, we evaluated the potential role of miR-155 in regulating DC immune responses. The current results suggest that increased NA may enhance DC immune response by increasing the levels of miR-155. Our research suggests that increased miR-155 enhances the DC immune responses induced by NA by increasing their ability to activate lymphocytes.

We found that over-expressed NA up-regulated the expression of CD80 and MHCII in cultured BMDCs, whereas the inhibition of endogenous miR-155 had the opposite effect. MiR-155 also modestly raised the expression of surface marker CD40; this effect was 

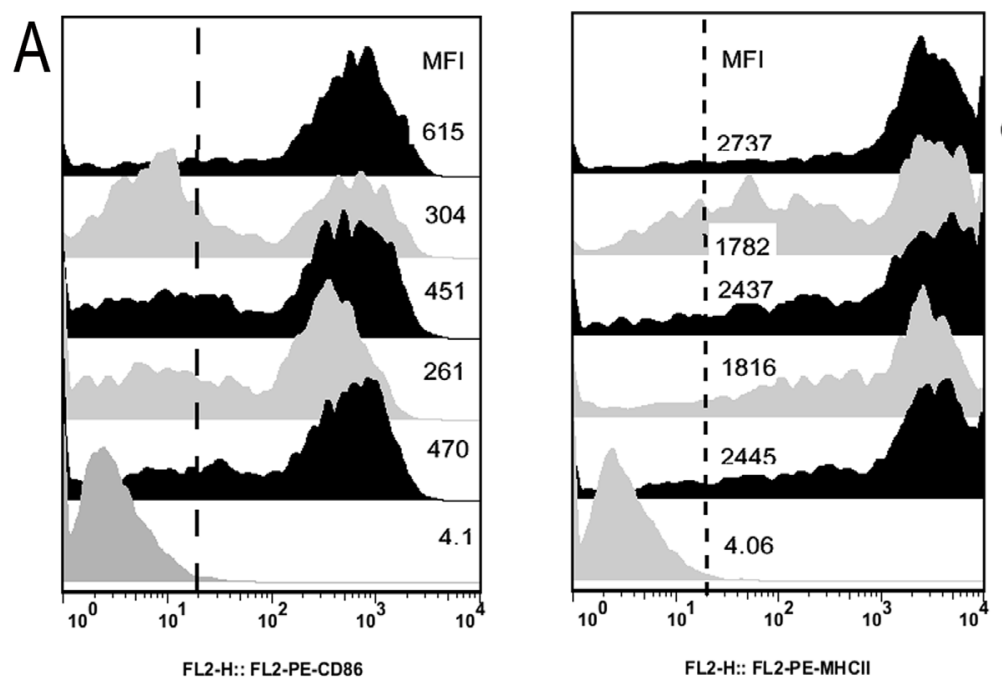

Ctrl-imyo1d+miR155

imyo1d+miR155

Ctrl-imyo1d

imyo1d

blank

isotype

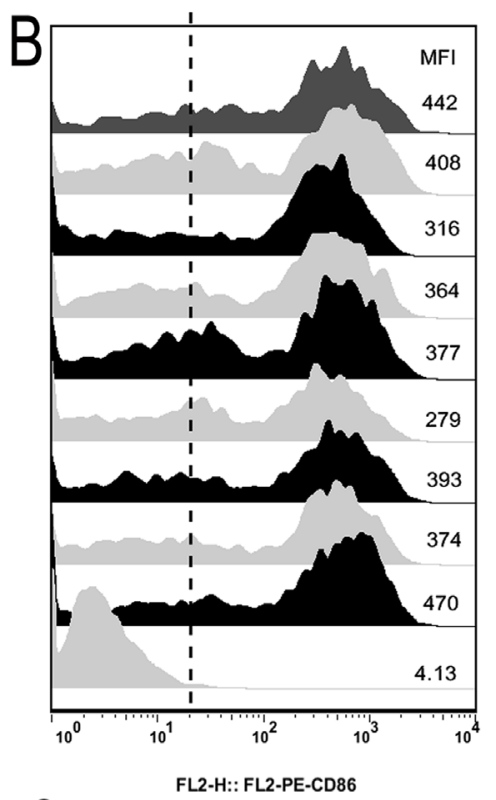

FL2-H:: FL2-PE-MHCII
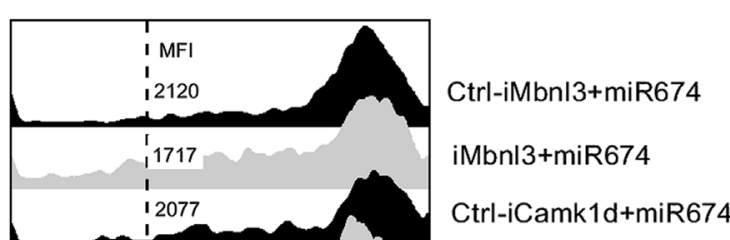

C
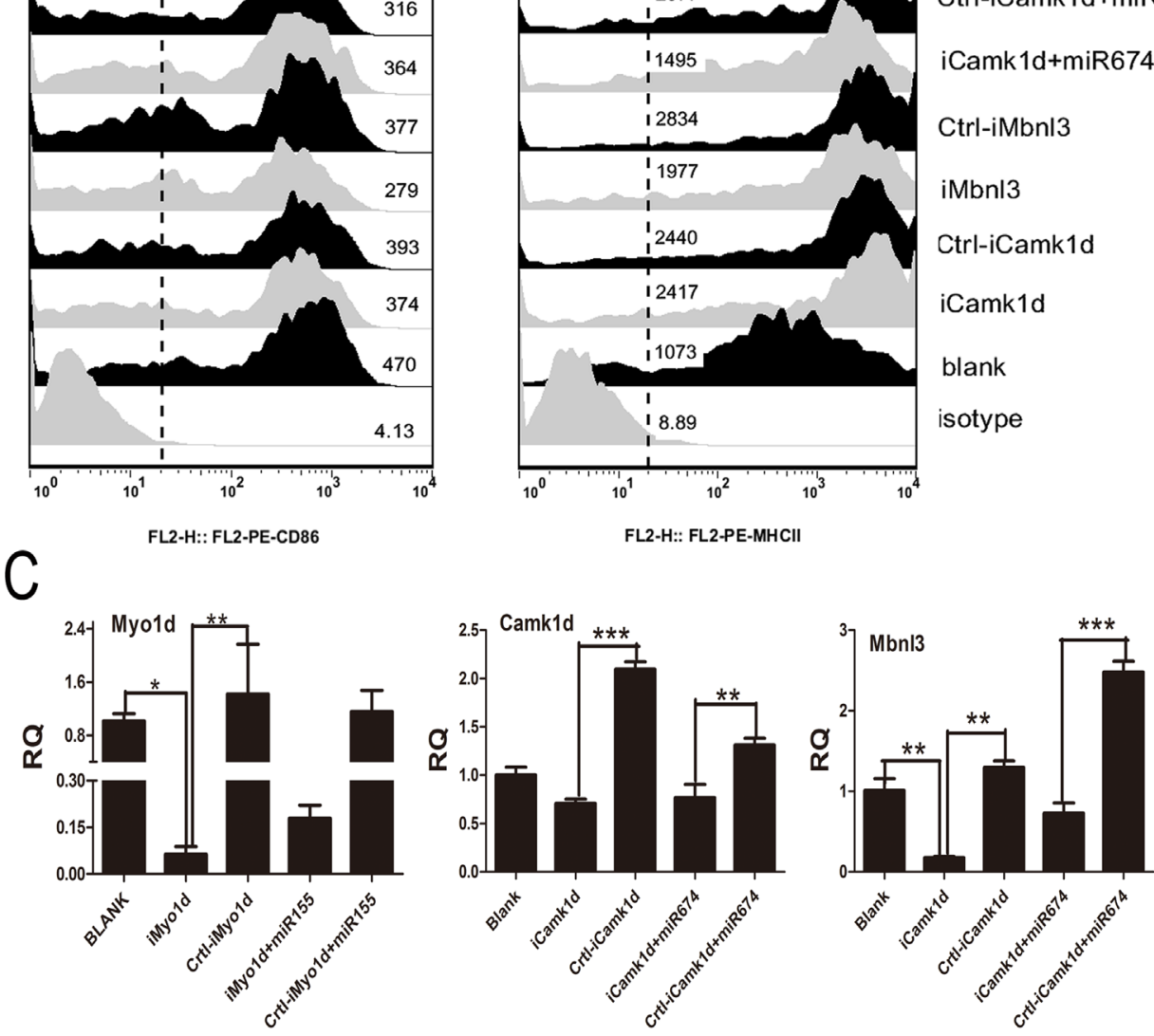

Figure 5: Results of target gene silencing treatment on DC phenotypic alterations. A. The expression of CD86 and MHCII on BMDCs when the target gene (Myold) of miR-155 was inhibited (DCs were divided into Isotype, Blank, iMyold, Crtl-iMold, iMyold/ miR155, and Crtl-Myold/miR155). B. The expression of CD86 and MHCII on BMDCs when the target genes (Camk1d and Mbnl3) of miR-674 were inhibited (DCs were divided into Isotype, Blank, iCamk1d, Crtl-iCamk1d, iMbnl3, Crtl-iMbnl3, iCamk1d/ miR674, CrtliCamk1d/ miR674, iMbnl3/ miR674, and Crtl-iMbnl3/ miR674). C. qPCR analysis of the RNA interference results of Myo1d, Camk1d, and Mbnl3 in the above treatments. 

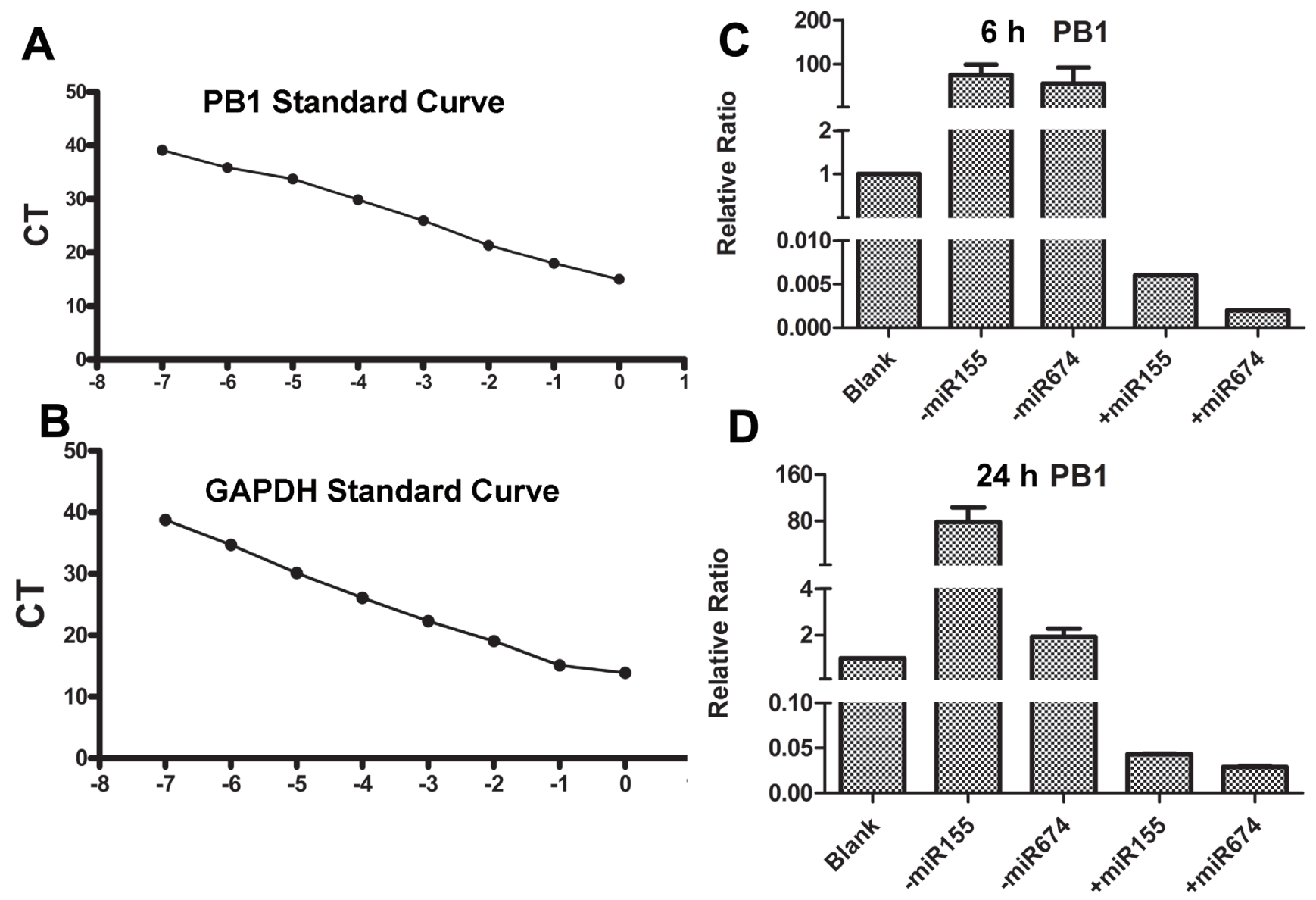

Figure 6: Detection of viral replication in DCs when miR155 and miR674 were inhibited or over-expressed. A. Standard curve for the PB1 gene from H9N2 AIV. B. Standard curve for the mouse GAPDH gene. C. Replication of H9N2 AIV following pretreatment of DCs with miRNAs for $6 \mathrm{~h}$. D. Replication of H9N2 AIV following pre-treatment of DCs with miRNAs for $24 \mathrm{~h}$.

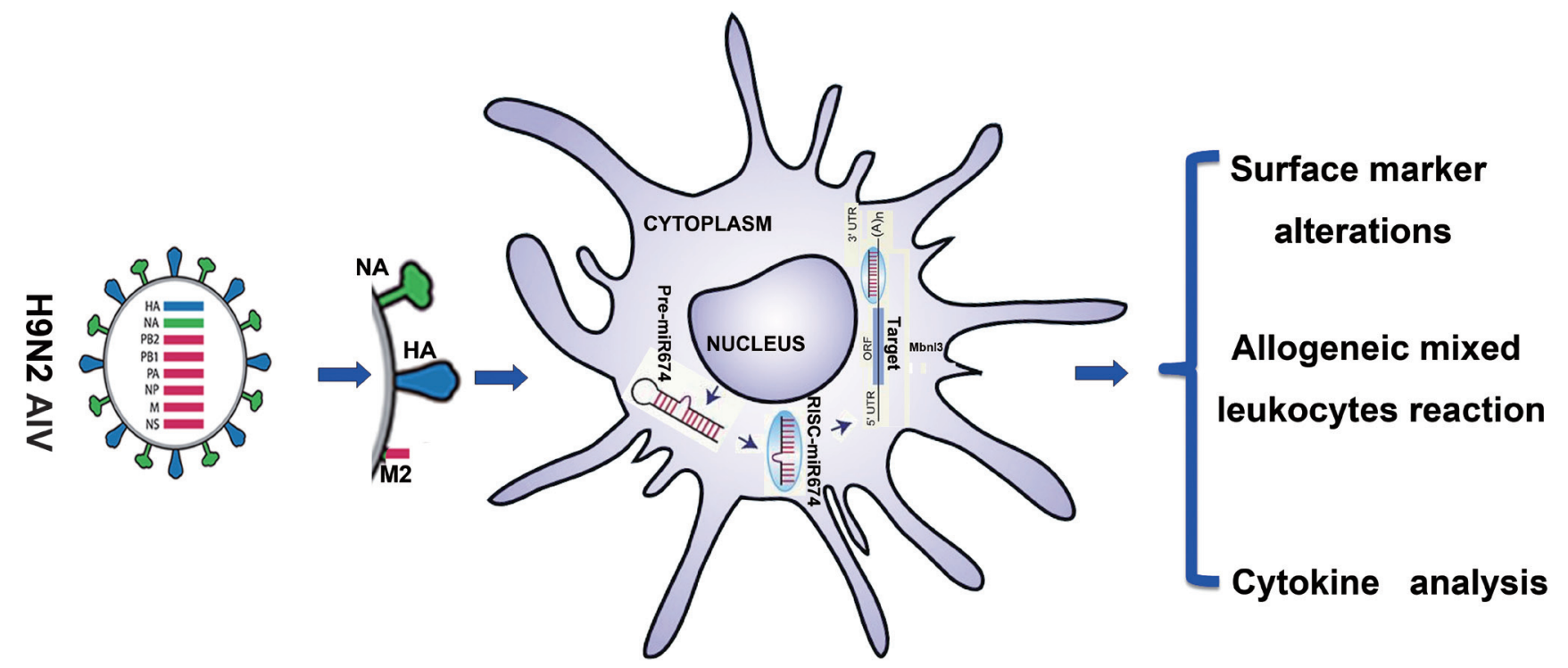

Figure 7: Summary of the research strategy. 
reversed by inhibiting endogenous miR-155. CD40 is a member of the TNF receptor family, which promotes the development of $\mathrm{T}$ cell immune responses by binding to CD40L [24]. Thus, miR-155 may enhance DC-T cell interactions by up-regulating DC surface maturation molecules. Moreover, increased NA or miR-155 also affected the ability of DCs to activate lymphocytes and secrete inflammatory cytokines. Our data revealed that NA and miR155 increased the expression of the proinflammatory cytokines IL-6, IL-10, and TNF- $\alpha$.

Compared with research on miR-155, studies on miR-674 are scarce. The current study is the first to focus on the role of miR-674 in evoking the immune activation of BMDCs. Functional experiments demonstrated that miR-674 significantly increased the percentage ratio and MFI of CD40- and CD80-positive cells in cultured BMDCs and that inhibition of the endogenous miR-674 reversed the effects. Moreover, miR-674 enhanced the ability of DCs to activate lymphocyte cells and secret cytokines, two additional standards for evaluating DC function [16]. Additionally, NA stimulation also promoted the above two functions. For cytokines, our data suggested that miR-674 increased the expression of IL-6, IL12 , and TNF- $\alpha$ but had no effect on anti-inflammatory cytokine IL-10. DCs promote the Th1 response via IL12 [25]. Accumulated IL-12 and enhanced proliferation of lymphocyte cells demonstrated that DCs matured and became activated when stimulated by miR- 674 .

Host defence against viral invasion involves activating the innate immune system with IFN- $\alpha$ [26]. The transcription of IFN- $\alpha$ is controlled primarily by members of the IFN regulatory factor family (specifically, IRF-3 and IRF-7) [27]. Viral infection results in the activation of IRF-3 and the low-level secretion of IFN- $\alpha$ on non-plasmacytoid DCs [28]. Previous research has demonstrated that virus protein NS1 activates IRF-3mediated IFN- $\alpha$ expression [29, 30]. Nevertheless, our data showed that IRF-3 was down-regulated in the NA and miR-674 treated group, indicating a repression of IFN- $\alpha$ production. This finding is consistent with our hypothesis that NA impacts DC functions by up-regulating miR-155 and miR-674.

Most genes targeted by miR-674 are involved in immune and inflammatory responses. Additionally, based on the identified genes, Pgm2l1, Entpd6, Aldh18a1, Camkld, Igflr, and Mbnl3 were especially distinctive in the gene interaction network. Our results showed that miR-674 repressed the expression of Pgm2l1, Aldh18a1, Camkld, and Mbnl3. Of these target genes, the silence of Mbnl3 could decrease the surface marker of CD86 and MHCII, which might represent a mechanism for regulating DCs. Signal pathway molecules and adaptor proteins are identified as strong miRNA targets. For example, TAK1-binding protein, targeted to miR155, is elevated to activate the $\mathrm{p} 38$ pathway [31]. TRAF6, a target of miR-146, is important for NF-kB activation [32]. Our data demonstrated that both NA and miR-155- or miR674-treated groups activated p38 pathways, which might be contributed to the regulation of IFN- $\alpha$ by decreasing IRF-3.

In summary, our results suggest that cellular miRNAs are important factors in regulating host DC functioning related to defending against viral infection and provide a deeper understanding of the mechanisms underlying the host defence system. We demonstrated a previously unidentified role for miR674 in the activation of immune responses. We suggest that miR674 mediates the NA-induced immune responses related to DCs by targeting and repressing Mbnl3 gene (Figure 7). Thus, miR-674 may be a new immune reinforcing agent that can be used in activating the DC function involved in defending against H9N2 AIV.

\section{MATERIALS AND METHODS}

\section{Virus and animals}

Influenza A virus (A/duck/Nanjing/01/1999(H9N2)) was provided by the Institute of Animal Husbandry and Veterinary Medicine, Jiangsu Academy of Agricultural Science (Nan Jing, China). Allantoic fluid was concentrated 10 -fold ( $10^{9}$ egg infectious doses 50 (EID50) / $0.1 \mathrm{ml}$ ) and purified on a discontinuous sucrose density gradient as described [33]. SPF C57BL/6 and BALB/c mice were obtained from Comparative Medical Center of Yang Zhou University.

\section{Ethics statement}

This study was approved by the Ethical Committee of Animal Experiments of the College of Veterinary Medicine, Nanjing Agricultural University. All animal care and use were conducted in strict accordance with the Animal Research Committee guidelines of the College of Veterinary Medicine, Nanjing Agricultural University.

\section{Plasmids and cell culture}

Four segments unrelated to viral replication (NA, HA, M2 and NS) were amplified from the H9N2 virus and cloned into pcDNA3.1 (Invitrogen). Primers were listed in Table 1. MiRNAs (miR-155, miR-674, miR-499 and miR-181b1) were amplified and cloned into pSilencer4.1 (Invitrogen), whose primers were listed in Table 2. The 3'-UTRs of Myold mRNAs target to miR-155 and the 3'UTRs of Pgm2l1, Aldh18a1, Entpd6, Camk1d, Igflr and Mbnl3 mRNAs, harboring the predicted miR-674 binding 
Table 1: Primers used in amplified NA, M2, NS and HA

\begin{tabular}{|l|l|l|}
\hline Gene & Sequence & Products \\
\hline NA Sence & GGGTACCATGAATCCAAATCAGAAG & \multirow{2}{*}{$\mathbf{1 4 1 8}$ bp } \\
\hline NA Anti-sence & GCTCGAGTTATATAGGCATGAAGTTG & \multirow{2}{*}{$\mathbf{7 7 3}$ bp } \\
\hline M2 Sence & GGGTACCATGAGTCTTCTAACCGAG & \multirow{2}{*}{$\mathbf{7 0 7}$ bp } \\
\hline M2 Anti-sence & GCTCGAGTCACTTGAATCGCTGC & \\
\hline NS Sence & GGGTACCATGGATTCCAACACTG & $\mathbf{1 6 8 3}$ bp \\
\hline HS Anti-sence & GCTCGAGTTAAATAAGCTGAAACGAG \\
\hline HA Anti-sence & GGGTACCATGGAAGTAGTATCACTAAT & GCTCGAGTTATATACAAATGTTGCATC \\
\hline
\end{tabular}

Table 2: Primers used in amplified miR155, miR674, miR181b1 and miR499

\begin{tabular}{|c|c|c|}
\hline Gene & Sequence & Products \\
\hline MiR155 Sence & GCGGGATCCTATTTCTTTTTCTCTTTG & \multirow[b]{2}{*}{485 bp } \\
\hline MiR155 Anti-sence & GCGAAGCTTACTGCTGATCTATCTTTTA & \\
\hline MiR181b1 Sence & GCGGGATCCATTCAAATAAAAACCATC & \multirow{2}{*}{504 bp } \\
\hline MiR181b1 Anti-sence & GCGAAGCTTTTAGTGACTTCCTCACAG & \\
\hline MiR674 Sence & GCGGGATCCTTAACTCAACTACCCAGGT & \multirow[b]{2}{*}{530 bp } \\
\hline MiR674 Anti-sence & GCGAAGCTTACAAAAATCCCCAAACAC & \\
\hline MiR499 Sence & GGGATCCCGCAAGAAAGCAGCATC & \multirow[b]{2}{*}{503 bp } \\
\hline MiR499 Anti-sence & GAAGCTTCACCCCAAACACCACCT & \\
\hline
\end{tabular}

sequences, were amplified and cloned into pMIR-Report luciferase vector (Ambion, TX) with primers listed in Table 3. The shRNA target to Myold, Camkld and Mbnl3 gene were designed and purchased from Invitrogen. Primers are list in Table 4. The artificial synthesis shRNA was then annealed and cloned into the BamHI and EcoRI sites of pLVX-shRNA1 using compatible restriction sites flanking the oligo siRNA target sequence. The expressing vector pLVX-shRNA1 expression vector and lentivirus transfection packaging kit (Lenti-X Packaging System) were purchased from Clontech (Mountain View, CA, USA).

Bone marrow-derived dendritic cells (BMDCs) were prepared from the femurs and tibias of sacrificed 4-6 wk old C57BL/6 mice as described [7]. At day 6, the nonadherent, relatively immature DCs were harvested for subsequent assays. 293 cells were cultured in Earle's modified Eagle's medium containing 10\% fetal bovine serum (HyClone), 100 units/ml penicillin, and $100 \mathrm{~g} /$ $\mathrm{ml}$ streptomycin at $37^{\circ} \mathrm{C}$ under $5 \% \mathrm{CO}_{2}$, and used for luciferase reporter assays. Cells were transfected using Lipofectame2000 (Invitrogen).

\section{MiRNAs selection and quantitative PCR validation}

Small RNAs were selected from our previous Microarray data [7]. The complete data set for each miRNA have been listed in supplement1. To test which viral protein mainly charged for the alteration of miRNAs, we amplified four segments (NA, HA,
M2 and NS) and then cloned into pcDNA3.1 vector. According to microarray result, 9 up-regulated and 8 down-regulated genes were selected for quantitative PCR (qPCR) verification. Small RNAs were purified using the miRNeasy mini kit (Qiagen), reverse transcribed using the miScript Reverse Transcriptase, and qPCR performed using the QuantiTect SYBR Green PCR master mix (Qiagen). miRNAs expression was normalized to the internal control 5S rRNA. Primers for the 17 selected miRNAs are list in Table 5. All assays were performed in triplicates. Relative expression levels were calculated using the 2- $\Delta \Delta \mathrm{Ct}$ method [34].

\section{Immune response of BMDCs stimulated by NA}

\section{Surface marker analysis of BMDCs}

Immature BMDCs were plated into fresh medium $\left(1 \times 10^{6}\right.$ cells $\left./ \mathrm{ml}\right)$ and transfected with constructed vector (NA, HA, M2 and NS), pcDNA3.1 (negative control) and LPS ( $1 \mu \mathrm{g} / \mathrm{ml}$, positive control) for $48 \mathrm{~h}$. Plasmids were transfected with lipofectame2000 reagent (Invitrogen). Then cells samples $\left(1 \times 10^{6}\right.$ cells, $1.5 \mathrm{ml}$ tube $)$ were collected, washed twice with PBS and incubated at $4^{\circ} \mathrm{C}$ for $30 \mathrm{~min}$ with the following monoclonal antibodies (anti-mouse CD11c, anti-mouse CD40, anti-mouse CD86, anti-mouse MHC class II and anti-mouse CD80 antibody, respectively). After washing, cells were analyzed with Fluorescence Activated Cell Sorter (FACS) (BD, FACS Aria). 
Table 3: Primers used in amplified miRNAs target genes

\begin{tabular}{|c|c|c|}
\hline Gene & Sequence & Products \\
\hline \multicolumn{3}{|l|}{ MiR155 targets } \\
\hline Myo1d Sence & ATAGAGCTCCAGCACCTGGTCTCCTAC & \multirow[b]{2}{*}{630 bp } \\
\hline Myo1d Anti-sence & GCAAGCTTAAGATTTAATGCTTTATTGCTC & \\
\hline \multicolumn{3}{|l|}{ MiR674 targets } \\
\hline Pgm2l1 Sence & ATAGAGCTCGCATGTACGGGACATAAC & \multirow{2}{*}{530 bp } \\
\hline Pgm2l1 Anti-sence & CAAGCTTTGCCACTGGAACACTTTC & \\
\hline Entpd6 Sence & ATAGAGCTCTGGAAGTGACACCATCCT & \multirow[b]{2}{*}{880 bp } \\
\hline Entpd6 Anti-sence & GCAAGCTTAAACCCTTGTAAACCTTTAT & \\
\hline Aldh18a1 Sence & ATAGAGCTCTCTTGGAAGGGTCTGTCTT & \multirow[b]{2}{*}{720 bp } \\
\hline Aldh18a1 Anti-sence & CAAGCTTCTGGAGGTCGAGAATAGG & \\
\hline Camk1d Sence & ATAGAGCTCCCTGCATAGGACTGGAAG & \multirow[b]{2}{*}{548 bp } \\
\hline Camk1d Anti-sence & GCAAGCTTCCTCTGGAAGAAGGGACT & \\
\hline Igf1r Sence & ATAGAGCTCCGCCAACAGTAACGTGT & \multirow[b]{2}{*}{690 bp } \\
\hline Igf1r Anti-sence & CAAGCTTCCAAACCGAAAACAGGAT & \\
\hline Mbnl3 Sence & ATAGAGCTCTAAGCTGGCACTCTAGTTG & $785 \mathrm{bp}$ \\
\hline Mbnl3 Anti-sence & CAAGCTTCTGTAGCTGCTGTTCGTC & \\
\hline
\end{tabular}

Table 4: Sequence of shRNA target to Myold, Camkld and Mbnl3 gene

\begin{tabular}{|c|c|}
\hline Gene & Sequence \\
\hline iMYO1D-1-F & gatcc GCTATTGCTGACGCTGCTTACCGAAGTAAGCAGCGTCAGCAATAGC TTTTTT g \\
\hline iMYO1D-1-R & aattc AAAAAA GCTATTGCTGACGCTGCTTACTTCGGTAAGCAGCGTCAGCAATAGC g \\
\hline iMYO1D-2-F & gatcc GCAGATGCTGCACTCTCTTCACGAATGAAGAGAGTGCAGCATCTGC TTTTTT g \\
\hline iMYO1D-2-R & aattc AAAAAA GCAGATGCTGCACTCTCTTCATTCGTGAAGAGAGTGCAGCATCTGC g \\
\hline Ctrl-iMYO1D-1-F & gatcc GATGTCGCAGATGTCGTAGACCGAAGTCTACGACATCTGCGACATC TTTTTT g \\
\hline Ctrl-iMYO1D-1-R & aattc AAAAAA GATGTCGCAGATGTCGTAGACTTCGGTCTACGACATCTGCGACATC g \\
\hline iCAMK1D -1-F & gatcc GGAAGCTCTTCGCAGTGAAGTCGAAACTTCACTGCGAAGAGCTTCC TTTTTT g \\
\hline iCAMK1D-1-R & aattc AAAAAA GGAAGCTCTTCGCAGTGAAGTTTCGACTTCACTGCGAAGAGCTTCC g \\
\hline iCAMK1D-2-F & gatcc GCAGCCTGGACAGTTCAAATGCGAACATTTGAACTGTCCAGGCTGC TTTTTT g \\
\hline iCAMK1D-2-R & aattc AAAAAA GCAGCCTGGACAGTTCAAATGTTCGCATTTGAACTGTCCAGGCTGC g \\
\hline Ctrl-iCAMK1D-1-F & gatcc GATGTCGCAGATGTCGTAGACCGAAGTCTACGACATCTGCGACATC TTTTTT g \\
\hline Ctrl-iCAMK1D-1-R & aattc AAAAAA GATGTCGCAGATGTCGTAGACTTCGGTCTACGACATCTGCGACATC g \\
\hline iMBNL3-1-F & gatcc GCACTCGTGAGAACTGCAAGTCGAAACTTGCAGTTCTCACGAGTGC TTTTTT $\mathrm{g}$ \\
\hline iMBNL3-1-R & aattc AAAAAA GCACTCGTGAGAACTGCAAGTTTCGACTTGCAGTTCTCACGAGTGC g \\
\hline iMBNL3-2-F & gatcc GCACTTAAAGTCGCAGCTAGACGAATCTAGCTGCGACTTTAAGTGC TTTTTT g \\
\hline iMBNL3-2-R & aattc AAAAAA GCACTTAAAGTCGCAGCTAGATTCGTCTAGCTGCGACTTTAAGTGC g \\
\hline Ctrl-iMBNL3-1-F & gatce AGTGCAGCAAGAGTGACTCTCCGAAGAGAGTCACTCTTGCTGCACT TTTTTT g \\
\hline Ctrl-iMBNL3-1-R & aattc AAAAAA AGTGCAGCAAGAGTGACTCTCTTCGGAGAGTCACTCTTGCTGCACT g \\
\hline
\end{tabular}

\section{Allogeneic mixed leukocytes reaction (MLR) proliferation assays}

The primary T-cell stimulatory capacity of BMDCs was examined in a MLR. Untreated and variously treated BMDCs (pcDNA3.1- stimulated, NA-stimulated and LPSstimulated $(100 \mathrm{ng} / \mathrm{ml}))$ were used as the stimulator cells. Allogeneic lymphocytes were obtained from BALB/c as follows. Leukocytes were isolated from the spleens of 4 to 6 week-old mice and cultured in complete RPMI 1640 medium supplemented with $10 \%$ FCS in 96-well plates at $37^{\circ} \mathrm{C}$ for $48 \mathrm{~h}$. Graded numbers of responder cells $\left(1 \times 10^{5}\right.$ cells/well) were added to $96-w e l l$ round bottomed plates, giving responder: stimulator ratios of $1: 1$ or $5: 1$, in a culture volume of $100 \mu \mathrm{L}$. Cell proliferation assays were conducted with the Cell Counting Kit-8 (CCK-8, Beyotime). Each well received $20 \mu \mathrm{L}$ CCK-8 solution and was incubated for a further $2 \mathrm{~h}$ at $37^{\circ} \mathrm{C}$ before absorbance measurement at $450 \mathrm{~nm}$. All experiments were conducted in triplicates. The Stimulation Index was calculated using the formula: $\mathrm{SI}=\left(\mathrm{OD}_{\text {sample }}-\mathrm{OD}_{\text {stimulator cells only }}\right) /\left(\mathrm{OD}_{\text {responder cells }}\right.$ only $-\mathrm{OD}_{\text {blank control }}$ [35]. 
Table 5: qRT-PCR primers used for detecting miRNAs alteration

\begin{tabular}{|l|l|l|}
\hline MiRNA & Mibase number & Sence primer \\
\hline mmu-miR-155-5p & MIMAT0000165 & GGGTTAATGCTAATTGTGATAGGGGT \\
\hline mmu-mir-680-1 & MI0004640 & CCCGTAGACGACTGTACCCCC \\
\hline mmu-miR-674-3p & MIMAT0003741 & GGGCACAGCTCCCATCTCAGAACA \\
\hline mmu-miR-222-5p & MIMAT0017061 & GGGTCAGTAGCCAGTGTAGATCCT \\
\hline mmu-miR-221-3p & MIMAT0000669 & GGGAGCTACATTGTCTGCTGGG \\
\hline mmu-miR-707 & MIMAT0003497 & CAGTCATGCCGCTTGCCTACG \\
\hline mmu-mir-680-2 & MI0004641 & CGGGCATCTGCTGACATGGGGG \\
\hline mmu-miR-22-3p & MIMAT0000531 & GGAAGCTGCCAGTTGAAGAACTGT \\
\hline mmu-miR-499-5p & MIMAT0003482 & GGGGTTAAGACTTGCAGTGATGTTT \\
\hline mmu-miR-375-3p & MIMAT0000739 & TTTGTTCGTTCGGCTCGCGT \\
\hline mmu-miR-29c-3p & MIMAT0000536 & GGCGTAGCACCATTTGAAATCG \\
\hline mmu-miR-146b-5p & MIMAT0003475 & GGGGTGAGAACTGAATTCCATAGGCT \\
\hline mmu-miR-687 & MIMAT0003466 & GGGCTATCCTGGAATGCAGCAATGA \\
\hline mmu-miR-24-1-3p & MIMAT0000219 & GGTGGCTCAGTTCAGCAGGAAC \\
\hline mmu-miR-339-5p & MIMAT0000584 & TCCCTGTCCTCCAGGAGCTCAC \\
\hline mmu-miR-181b-5p & MIMAT0000673 & AACATTCATTGCTGTCGGTGGGT \\
\hline mmu-miR-679-3p & MIMAT0017248 & GGAGCAAGGTCCTCCTCACAGTAG \\
\hline
\end{tabular}

Table 6: qRT-PCR primers used for detecting target genes and viral segments

\begin{tabular}{|l|l|l|}
\hline Gene name & Sence primer & Anti-Sence primer \\
\hline Pgm211 & cctccaattccagtcccaga & atccaccacccaaacaaagc \\
\hline Aldh18a1 & tgtaatgccetggagacgtt & gacttcacttctgaggggct \\
\hline Camk1d & tccgactctgccaaagactt & acgcttgtctccatttgctc \\
\hline Mbn13 & gctatgctcaccctacggat & gatgagctgccctgagtttg \\
\hline MYO1D & attcgaacacccegtacact & ttggccacttcacatgcttc \\
\hline NA & GCAGAGACAATTGGAAGGGC & CATTCCCATCGTCAAAGGCC \\
\hline HA & TGGGAAGGGATGCTTCGAAT & CATGGCCCAGAACATGAAGG \\
\hline NS & TCCTTCCCGAGTAGCAGTTC & ACTGTGTCAAGCTTCCAGGT \\
\hline M2 & AGATGGCGACTACCACCAAC & AGTCCCAATTGTCCTCATCG \\
\hline
\end{tabular}

\section{Cytokine measurement}

BMDC culture supernatants were collected at $24 \mathrm{~h}$ after treatments (Groups were divided as MLR experiments). Concentrations of TNF- $\alpha$, IL-6, IL-10 and IL-12p70 in the supernatants were measured using the Quantikine Elisa kit (Boster). The sensitivity of the assay was $2 \mathrm{pg} / \mathrm{ml}$ for TNF- $\alpha, 4 \mathrm{pg} / \mathrm{ml}$ for IL-6, IL-10 and IL$12 \mathrm{p} 70$.

\section{qRT-PCR validation}

BMDCs was cultured and collected at $24 \mathrm{~h}$ after treatments with NA segment. Then, qPCR was conducted to determine the alteration of Pgm211, Aldh18a1, Camk1d and Mbnl3, targets of miR674 or miR155. Meantime, we also evaluated the transcription efficient of plasmid pcDNA3.1-NA, M2, NS and HA by qPCR. Primers were all listed in Table 6.

\section{Immune response of BMDCs stimulated by miRNAs}

\section{Plasmid construction and phenotypic detection}

To confirm the phenotype alteration induced by NA segment may meditated by miRNAs. MiRNAs overexpression vector were constructed based on pSilencer4.1 vector (Invitrogen). Four selected miRNAs (miR155, miR499, miR674 and miR181b1) were amplified and then cloned into pSilencer4.1, whose primers were listed in Table 2. The isolation of BMDCs and phenotypic detection were as previous description.

\section{MLR proliferation assays, Cytokines analysis and qRT-PCR validation}

Cells were grouped as non-stimulated, pSilencer4.1stimulated (negative control), miR155- or miR674stimulated groups and LPS-stimulated (positive control). MLR detection was performed as previous. Moreover, cells culture supernatants were collected at $24 \mathrm{~h}$ for 
Table 7: The predicted genes target position and mutated position of miR155 and miR674

\begin{tabular}{|c|c|c|}
\hline Gene & Sequence & Position \\
\hline Myo1d-mut & 5'...CAUCAAGAGCAAUAAATCTAGCAA... & \\
\hline Myo1d 3' UTR & 5'...CAUCAAGAGCAAUAAAGCAUUAA... & \multirow{2}{*}{$1922-1929$} \\
\hline mmu-miR-155 & 3'...UGGGGAUAGUGUUAAUCGUAAUU... & \\
\hline Pgm2L1-mut & $5^{\prime} \ldots$...AUUCUUGUCAAAUUCCTCTAGGC... & \\
\hline Pgm2L1 3' UTR & 5'...AUUCUUGUCAAAUUCCUCAGUGC... & 618-624 \\
\hline mmu-miR-674 & 3'...AUGUGGUGAGGGUAGAGUCACG... & \\
\hline Entpd6-mut & 5'...GCUUCCCCAUGGCCCCTCTAGGA... & \\
\hline Entpd6 3' UTR & 5'...GCUUCCCCAUGGCCCCUCAGUGA... & 848-855 \\
\hline mmu-miR-674 & 3'...AUGUGGUGAGGGUAGAGUCACG & \\
\hline Aldh18a1-mut & $5^{\prime}$ '...UGGCUGCCGGAACGCCTCTAGGA... & \\
\hline Aldh18a1 3' UTR & $5^{\prime}$ '...UGGCUGCCGGAACGCCUCAGUGA... & $416-423$ \\
\hline mmu-miR-674 & 3'...AUGUGGUGAGGGUAGAGUCACG... & \\
\hline Camk1d -mut & 5'...CACCACUUCCGCUCUCTCTAGGU... & \\
\hline Camk1d 3' UTR & 5'...CACCACUUCCGCUCUCUCAGUGU... & 305-311 \\
\hline mmu-miR-674 & 3'...AUGUGGUGAGGGUA---GAGUCACG... & \\
\hline IGF1R -mut & 5'...ACAAGCCUCCUGUACCTCTAGGG... & \\
\hline IGF1R 3' UTR & 5'...ACAAGCCUCCUGUACCUCAGUGG... & 115-121 \\
\hline mmu-miR-674 & 3'...AUGUGGUGAGGGUA--GAGUCACG... & \\
\hline Mbnl3 -mut & 5'...AUCUUCCUAAAGAGGCTCTAGGG... & \\
\hline Mbnl3 3' UTR & 5'...AUCUUCCUAAAGAGGCUCAGUGG... & $9327-9333$ \\
\hline mmu-miR-674 & 3'...AUGUGGUGAGGGUAGAGUCACG... & \\
\hline
\end{tabular}

cytokines detection. The concentrations of TNF- $\alpha$, IL6 , IL-10 and IL-12p70 were measured as previous. Furthermore, BMDCs was cultured and collected at 24 $\mathrm{h}$ after treatments with miR674 or miR155. Then, qPCR was conducted to determine the alteration of Pgm211, Aldh18a1, Camk1d and Mbnl3, targets of miR674 or miR155.

\section{MiRNAs inhibiting experiment}

To detect whether the phenotypic alteration of BMDCs induced by NA was mediated by miRNAs, miRNAs inhibitors were designed and purchased from RIBBIO (Guangzhou, China). Each $100 \mathrm{nM}$ miRNAs inhibitors (miR155, miR499, miR674 and miR181b1) were transfected into BMDCs for $2 \mathrm{~h}$, before NA overexpression plasmid was transfected. After another $24 \mathrm{~h}$, BMDCs were collected for phenotypic detection with FACS.

\section{MiRNA target prediction and validation}

Target genes of miR-155 and miR-674 were predicted using miRanda and Targetscan [36]. Only target genes identified by these two algorithms were considered. Besides, we matched predicted genes with our previous microarray data to narrow the target. The 3'UTRs of selected target genes (Myold, Pgm2ll, Aldh18al,
Entpd6, Camkld, Igflr and Mbnl3), harboring the predicted miR155 and miR674 binding sequences, were amplified and cloned into pMIR-Report luciferase vector (Ambion, TX) (Table 3). Meantime, the mutated vector was constructed by overlap PCR methods. The predicted genes target position and mutated position of miR155 and miR674 was listed in Table 7. To determine whether miR-155 and miR-674 could repress the expression of target genes, $293 \mathrm{~T}$ cells were transfected with miR-155 or miR-674 and pMIR-Report vector, along with pRL-TK to normalize transfection efficiencies. Luciferase assays were performed using the dual-luciferase reporter assay system kit (Promega), according to the manufacturer's protocol, on a Modulus single-tube multimode reader (Promega).

\section{Phenotypic analysis of target genes shRNA- transfected DCs}

\section{Construction of recombinant lentivirus vectors}

To confirm whether miR-155 and miR-674 activated the immune function of DCs by inhibiting their target genes. The knockdown of Myold, Camkld and Mbnl3 in BMDCs was performed by RNA interference (RNAi) based on vector pLVX-shRNA. The targeting sequence of shRNA against Myold, Camk1d and Mbnl3 were designed using online design tools (http://rnaidesigner. lifetechnologies.com/rnaiexpress/design.do; Block-iT RNAi Designer). Six pairs of siRNA oligonucleotides 
Table 8: Primers used in the absolute $q$ RT-PCR for detecting virus replication

\begin{tabular}{|l|l|l|}
\hline Gene & Sequence & Products \\
\hline PB1 Sence & AGCGGGTATGCACAAACAGA & \multirow{2}{*}{$\mathbf{1 5 0}$ bp } \\
\hline PB1 Anti-sence & ATAAGTCTGGCGACCTTGGG & \multirow{2}{*}{$\mathbf{2 2 3}$ bp } \\
\hline GAPDH Sence & AACTTTGGCATTGTGGAAGG & \\
\hline GAPDH Anti-sence & ACACATTGGGGGTAGGAACA &
\end{tabular}

Table 9: Primers used in amplifying Myo1d, Caml1d and Mbnl3

\begin{tabular}{|c|c|c|}
\hline Gene & Sequence & Products \\
\hline Myo1d Forword & GGGTACCatggeggagcaggagagce & \multirow[b]{2}{*}{ 3034bp } \\
\hline Myo1d reverse & GCTCGAGtcagttccegggcacactg & \\
\hline Camk1d Forword & GGGTACCatggeccgggagaacggc & \multirow{4}{*}{$1160 \mathrm{bp}$} \\
\hline Camk1d reverse & GCTCGAGtcacttgettceagtgtgec & \\
\hline Mbnl3 Forword & GGGTACCatgacacctgtcaatgtagc & \\
\hline Mbnl3 reverse & GGGTACCtcaatatttcaactggttgectg & \\
\hline
\end{tabular}

targeting 3 target genes were designed and synthesized by Invitrogen, which were listed in Table 4 . The annealed oligonucleotides were cloned into the BamHI and EcoRI sites of pLVX-shRNA1 using compatible restriction sites flanking the oligo siRNA target sequence. We also generated a mutated siRNA control construct for each target gene, which is not predicted to target any genes (Table 4). All clones were verified by DNA sequencing.

\section{Lentivirus packaging}

The pseudotyped lentiviruses were produced in $293 \mathrm{~T}$ cells by co-transfection with the recombinant siRNA plasmids (siMyold-1, siMyold-3, Ctrl-siMyo1d-5, siCamk1d-7, siCamk1d-9, Ctrl-siCamk1d-11, siMbnl-13, siMbnl-15 and Ctrl-siMbnl-17) and the three Clontech Lenti-X HT packaging vectors. After $48 \mathrm{~h}$ of transfection, the cell supernatants were collected and centrifuged at $500 \times \mathrm{g}$ for $10 \mathrm{~min}$; the soluble supernatant fractions containing the lentiviruses were collected and stored for later using.

\section{Phenotypic analysis of shRNA-transfected DCs}

BMDCs were transfected with siMyo1d, siCamk1d, siMbnL3 and their Ctrl-siRNAs at $37^{\circ}$ in $5 \% \mathrm{CO} 2$ for $48 \mathrm{~h}$. Then, BMDCs were collected to detect the RNA interference efficiency by qPCR. Then BMDCs were infected with the effective interference plasmid and part of cells were subsequently stained with phycoerythrinor fluorescein isothiocyanate-conjugated monoclonal antibodies (mAbs) against mouse CD40, CD80, CD86 and mouse MHC class II molecules for $30 \mathrm{~min}$ at $4^{\circ} \mathrm{C}$. Negative controls were isotype-matched mAbs. After washing, the cells were suspended and examined by FACS. Furthermore, the other part of DCs was transfected with miR155 or miR674 after target genes were silenced for $24 \mathrm{~h}$. Finally, this part of BMDCs was stained and examined by FACS.

\section{Western blot assay}

Since DCs were the only bridge communicated the innate and acquired immunity, we tried to evaluated how NA segment and their induced miRNAs affect the TLR and NF-kB signal pathways by western blot. BMDCs were transfect with NA and miR155 or miR674 over expression plasmid for $24 \mathrm{~h}$. Then cells were collected and washed with PBS three times for the next experiments. Western blot detection was performed as our previous article[7]. Mouse IkBa, P-IkBa, P38, P-P38, ERK, P-ERK, JUK, P-JUK, IRF-3, IRF-7, TRAF-3 and TRAF-6 were selected and detected according to each manufacturer's protocol. Protein bands were visualized using the Super ECL Plus system. GAPDH was used as a loading control.

\section{Virus replication experiments}

DCs were seeded onto 6 -well plates $\left(5 \times 10^{5}\right.$ cells /well) and pretreated with miRNAs inhibition or overexpression plasmid for $24 \mathrm{~h}$. Then, pretreated DCs were inoculated with $100 \mu \mathrm{L} \mathrm{H9N} 2 \mathrm{AIV}$ for $1 \mathrm{~h}$ at $37^{\circ} \mathrm{C}$ (Virus was diluted in PBS with $10^{6} \mathrm{TCID}_{50} / 100 \mu \mathrm{L}$ ). After that, DCs were washed five times with $1000 \mathrm{rmp} / 5 \mathrm{~min}$ to eliminate the non-absorbed virus and resuspended in fresh RPMI 1640 medium. After another 24 h, BMDCs were collected and total RNA was extracted with trizol reagents. RT-PCR was then performed according to instructions (TaKaRa). For real-time PCR, 7500 Real-Time PCR System (ABI) and SYBR Green Master (Takara) were used. Virus PB1 gene was choose as the target gene and the mouse GAPDH gene was used as the internal parameter (primers were list in Table.8). Quantification of the target gene was determined by relative standard curves. PB1 and GAPDH plasmids were used to produce a double-standard curve. The mouse GAPDH quantity at each sampling time was taken as the standard to determine 
the target genes quantity.

\section{Statistical analysis}

Data were evaluated by unpaired two-tailed Student's t-test using GraphPad Prism 5 (http://www. graphpad.com) (CSSN), with $\mathrm{p}$ values $<0.05$ considered to be statistically significant. The significance of the data was also determined by one-way ANOVA, followed by Tukey's multiple comparison tests. FACS data were analysed by FlowJo software (FlowJo, China). All data are expressed as mean \pm standard error of the mean.

\section{ACKNOWLEDGMENTS}

Authors would like to thank Prof. Xiankui Zhang (Medical University of South Carolina) and Prof. Yang Zeng (Nanjing agricultural University) for their insightful suggestions. This work was supported by the National Science Grant of P. R. China (No. 31172302), the National Science Grant of Jiangsu Province (No. BK20150666) and A Project Funded by the Priority Academic Program Development of Jiangsu Higher Education Institutions (PAPD).

\section{CONFLICTS OF INTEREST}

There is no conflict of interest.

\section{REFERENCES}

1. Gu H, Chen B, Zhu H, Jiang T, Wang X, Chen L, Jiang Z, Zheng D and Jiang J. Importance of Internet surveillance in public health emergency control and prevention: evidence from a digital epidemiologic study during avian influenza A H7N9 outbreaks. J Med Internet Res. 2014; 16:e20.

2. Wang D, Yang L, Gao R, Zhang X, Tan Y, Wu A, Zhu W, Zhou J, Zou S, Li X, Sun Y, Zhang Y, Liu Y, Liu T, Xiong $\mathrm{Y}, \mathrm{Xu}$ J, et al. Genetic tuning of the novel avian influenza $\mathrm{A}(\mathrm{H} 7 \mathrm{~N} 9)$ virus during interspecies transmission, China, 2013. Euro Surveill. 2014; 19.

3. Moscona A. Neuraminidase inhibitors for influenza. N Engl J Med. 2005; 353:1363-1373.

4. Eckard L and Webby R. Neuraminidase: another piece of the influenza vaccine puzzle. J Infect Dis. 2015; 212:11801 .

5. Rate A, Bosco A, McKenna KL, Holt PG and Upham JW. Airway epithelial cells condition dendritic cells to express multiple immune surveillance genes. PloS one. 2012; 7:e44941.

6. Qin T, Yin Y, Wang X, Liu H, Lin J, Yu Q and Yang Q. Whole inactivated avian Influenza H9N2 viruses induce nasal submucosal dendritic cells to sample luminal viruses via transepithelial dendrites and trigger subsequent DC maturation. Vaccine. 2015; 33:1382-1392.

7. Lin J, Yin YY, Qin T, Zhu LQ, Yu QH and Yang Q. Enhanced immune response of BMDCs pulsed with H9N2 AIV and CpG. Vaccine. 2014; 32:6783-6790.

8. Marcelin G, Sandbulte MR and Webby RJ. Contribution of antibody production against neuraminidase to the protection afforded by influenza vaccines. Rev Med Virol. 2012; 22:267-279.

9. Riberdy JM, Flynn KJ, Stech J, Webster RG, Altman JD and Doherty PC. Protection against a lethal avian influenza A virus in a mammalian system. J Virol. 1999; 73:14531459.

10. Lu X, Renshaw M, Tumpey TM, Kelly GD, Hu-Primmer $\mathrm{J}$ and Katz JM. Immunity to influenza A H9N2 viruses induced by infection and vaccination. J Virol. 2001; 75:4896-4901.

11. Hussain $M$ and Asgari S. MicroRNA-like viral small RNA from Dengue virus 2 autoregulates its replication in mosquito cells. Proc Natl Acad Sci U S A. 2014; 111:27462751.

12. Ma YJ, Yang J, Fan XL, Zhao HB, Hu W, Li ZP, Yu GC, Ding XR, Wang JZ, Bo XC, Zheng XF, Zhou Z and Wang SQ. Cellular microRNA let-7c inhibits M1 protein expression of the H1N1 influenza A virus in infected human lung epithelial cells. J Cell Mol Med. 2012; 16:2539-2546.

13. Smyth LA, Boardman D, Tung S, Lechler R and Lombardi G. MicroRNAs affect dendritic cells function and phenotype. Immunology. 2014.

14. Rosenberger CM, Podyminogin RL, Navarro G, Zhao GW, Askovich PS, Weiss MJ and Aderem A. miR-451 regulates dendritic cell cytokine responses to influenza infection. J Immunol. 2012; 189:5965-5975.

15. Bright RA, Neuzil KM, Pervikov Y and Palkonyay L. WHO meeting on the role of neuraminidase in inducing protective immunity against influenza infection, Vilamoura, Portugal, September 14, 2008. Vaccine. 2009; 27:6366-6369.

16. Banchereau J, Briere F, Caux C, Davoust J, Lebecque S, Liu YJ, Pulendran B and Palucka K. Immunobiology of dendritic cells. Annu Rev Immunol. 2000; 18:767-811.

17. Smyth LA, Boardman DA, Tung SL, Lechler R and Lombardi G. MicroRNAs affect dendritic cell function and phenotype. Immunology. 2015; 144:197-205.

18. Halbherr SJ, Ludersdorfer TH, Ricklin M, Locher S, Berger Rentsch M, Summerfield A and Zimmer G. Biological and protective properties of immune sera directed to the influenza virus neuraminidase. J Virol. 2015; 89:1550-1563.

19. Yamaya M, Nadine L, Kubo H, Saito K, Saito R and Nishimura $\mathrm{H}$. The effects of neuraminidase inhibitors on the release of oseltamivir-sensitive and oseltamivir-resistant influenza viruses from primary cultures of human tracheal epithelium. J Med Virol. 2015; 87:25-34.

20. Kamio N, Imai K, Shimizu K, Cueno ME, Tamura M, Saito $\mathrm{Y}$ and Ochiai K. Neuraminidase-producing oral mitis group streptococci potentially contribute to influenza viral 
infection and reduction in antiviral efficacy of zanamivir. Cell Mol Life Sci. 2015; 72:357-366.

21. Tucci M, Stucci S, Passarelli A, Giudice G, Dammacco F and Silvestris F. The immune escape in melanoma: role of the impaired dendritic cell function. Expert Rev Clin Immunol. 2014; 10:1395-1404.

22. Elton TS, Selemon H, Elton SM and Parinandi NL. Regulation of the MIR155 host gene in physiological and pathological processes. Gene. 2013; 532:1-12.

23. Seddiki N, Brezar V, Ruffin N, Levy Y and Swaminathan S. Role of miR-155 in the regulation of lymphocyte immune function and disease. Immunology. 2014; 142:32-38.

24. Gerlach AM, Steimle A, Krampen L, Wittmann A, Gronbach K, Geisel J, Autenrieth IB and Frick JS. Role of CD40 ligation in dendritic cell semimaturation. BMC Immunol. 2012; 13:22.

25. de Jong EC, Vieira PL, Kalinski P, Schuitemaker JH, Tanaka Y, Wierenga EA, Yazdanbakhsh $\mathrm{M}$ and Kapsenberg ML. Microbial compounds selectively induce Th1 cellpromoting or Th2 cell-promoting dendritic cells in vitro with diverse th cell-polarizing signals. J Immunol. 2002; 168:1704-1709.

26. Biron CA. Role of early cytokines, including alpha and beta interferons (IFN-alpha/beta), in innate and adaptive immune responses to viral infections. Semin Immunol. 1998; 10:383-390.

27. Taniguchi $\mathrm{T}$ and Takaoka A. The interferon-alpha/beta system in antiviral responses: a multimodal machinery of gene regulation by the IRF family of transcription factors. Curr Opin Immunol. 2002; 14:111-116.

28. Diebold SS, Montoya M, Unger H, Alexopoulou L, Roy P, Haswell LE, Al-Shamkhani A, Flavell R, Borrow P and Reis e Sousa C. Viral infection switches non-plasmacytoid dendritic cells into high interferon producers. Nature. 2003; 424:324-328.

29. Tisoncik JR, Billharz R, Burmakina S, Belisle SE, Proll SC, Korth MJ, Garcia-Sastre A and Katze MG. The NS1 protein of influenza A virus suppresses interferon-regulated activation of antigen-presentation and immune-proteasome pathways. J Gen Virol. 2011; 92:2093-2104.
30. Wang X, Li M, Zheng H, Muster T, Palese P, Beg AA and Garcia-Sastre A. Influenza A virus NS1 protein prevents activation of NF-kappaB and induction of alpha/beta interferon. J Virol. 2000; 74:11566-11573.

31. Broglie P, Matsumoto K, Akira S, Brautigan DL and Ninomiya-Tsuji J. Transforming growth factor betaactivated kinase 1 (TAK1) kinase adaptor, TAK1-binding protein 2, plays dual roles in TAK1 signaling by recruiting both an activator and an inhibitor of TAK1 kinase in tumor necrosis factor signaling pathway. J Biol Chem. 2010; 285:2333-2339.

32. Paik JH, Jang JY, Jeon YK, Kim WY, Kim TM, Heo DS and Kim CW. MicroRNA-146a downregulates NFkappaB activity via targeting TRAF6 and functions as a tumor suppressor having strong prognostic implications in NK/T cell lymphoma. Clin Cancer Res. 2011; 17:4761-4771.

33. Hartshorn KL, Crouch EC, White MR, Eggleton P, Tauber AI, Chang D and Sastry K. Evidence for a protective role of pulmonary surfactant protein D (SP-D) against influenza A viruses. J Clin Invest. 1994; 94:311-319.

34. Czescik A, Trzcinska A, Dunal-Szczepaniak $M$ and Siennicka J. [The use of real-time RT-PCR method for the determination of Toll-like genes expression at mRNA level]. [Article in Polish]. Med Dosw Mikrobiol. 2014; 66:17-22.

35. Liang J, Fu J, Kang H, Lin J, Yu Q and Yang Q. The stimulatory effect of TLRs ligands on maturation of chicken bone marrow-derived dendritic cells. Vet Immunol Immunopathol. 2013; 155:205-210.

36. Yan Z, Xiong Y, Xu W, Li M, Cheng Y, Chen F, Ding $\mathrm{S}, \mathrm{Xu} \mathrm{H}$ and Zheng $\mathrm{G}$. Identification of recurrence-related genes by integrating microRNA and gene expression profiling of gastric cancer. Int J Oncol. 2012; 41:2166-2174. 